\title{
DESENVOLVIMENTO E APLICAÇÃO EXPERIMENTAL DE CONTROLADORES FUZZY E CONVENCIONAL EM UM BIOPROCESSO
}

\author{
Manuela Souza Leite* \\ sl.manuela@gmail.com \\ Ana Maria Frattini Fileti* \\ Flávio Vasconcelos da Silva* \\ flaviolfeq.unicamp.br \\ *Laboratório de Controle e Automação de Processos, Faculdade de Engenharia Química, \\ Universidade Estadual de Campinas \\ Caixa Postal 6066 \\ CEP 13083-970 - Campinas, SP, Brasil
}

\section{RESUMO}

Este trabalho propõe a realização de um estudo comparativo do desempenho de controladores Fuzzy e convencional PID aplicados ao controle de temperatura de um processo de precipitação de bromelina do extrato aquoso de resíduos de abacaxi. Uma análise quantitativa da não-linearidade do processo foi realizada baseada na metodologia de curva de reação, aplicada em diferentes momentos da batelada, caracterizando o sistema por possuir diferente sensibilidade às ações de controle ao longo do tempo. O controlador convencional foi sintonizado a partir da aplicação das equações de Ziegler-Nichols aos parâmetros do processo obtidos nos instantes iniciais do experimento, seguido de sintonia fina por tentativa-e-erro. A sintonia do controlador Fuzzy consistiu na alteração do universo de discurso, na base de regras e na disposição das funções de pertinência, utilizando-se para isto o conhecimento obtido na análise das curvas de reação obtidas. Foi observado um melhor desempenho do controlador Fuzzy, apresentando menor valor da integral de erro absoluto multiplicado pelo tempo (ITAE), maior recuperação de atividade enzimática e menor consumo de energia elétrica para o resfriamento do sistema.

PALAVRAS-CHAVE: Controlador Fuzzy, controlador PID, Bromelina, Fieldbus.

\begin{abstract}
Development and Experimental Application of Fuzzy and Conventional Controllers to a Bioprocess

The present work focuses on a comparative study of the performance of Fuzzy and conventional PID controllers applied to temperature control in the bromelain precipitation process from aqueous extract of pineapple wastes. A quantitative analysis of process nonlinearities was performed based on process reaction curves methodology. Different sensitivity to control actions was detected at different batch times. The conventional controller was tuned by applying ZieglerNichols equations to the process parameters obtained at the beginning of the experiment, followed by fine tuning based on trial-and-error procedure. Using the knowledge obtained from the reaction curves analysis, the Fuzzy controller tuning consisted in changing the universe of discourse, the rule base and the support sets of membership functions. It was observed that the Fuzzy controller outperformed the PID, showing the smallest value of integral of time multiplied by absolute of the error (ITAE), a great recovery of enzymatic activity and the smallest consumption of electric energy for the system refrigeration.
\end{abstract}

KEYWORDS: Fuzzy controller, PID controller, Bromelain, Fieldbus. 


\section{INTRODUÇÃO}

O desenvolvimento de técnicas de Inteligência Artificial (IA) nos últimos anos ocupa cada vez mais posição de destaque em pesquisas na área de controle de processos industriais e, aos poucos, começam a ser utilizadas em plantas industriais com sucesso, como é o caso do controle fuzzy.

Observa-se que o controle clássico apresenta limitações de desempenho quando o processo apresenta alta complexidade, onde não-linearidades e o comportamento transiente estão presentes, como por exemplo, bioprocessos em batelada. Ao contrário, o controle fuzzy pode ser aplicado a sistemas lineares e não-lineares. É de fácil implementação, possui facilidades quanto à adequação de estratégia de controle humano, ao protocolo de controle, à simplicidade das leis de controle e à flexibilidade das variáveis lingüísticas, além de apresentar características de robustez (Shaw e Simões, 1999).

Muitos trabalhos envolvendo a aplicação de controladores fuzzy têm indicado que esta técnica é uma importante ferramenta para processos complexos (Lee, 1990). Aplicações práticas de lógica fuzzy em processos têm sido reportadas na literatura (Steyer et alli, 1997; Martínez et alli, 1999; Souza Jr. e Almeida, 2001; Babuska et alli, 2002; Horiuchi e Kishimoto, 2002; Antunes et alli, 2005; Silva, et alli, 2006, Fileti et alli, 2007).

Apesar da automação e o controle de processos influenciarem significativamente na diminuição dos custos de produção e na qualidade final dos bioprodutos em geral, ainda é tímido o número de trabalhos experimentais com aplicações práticas de controladores automáticos em processos de química fina. A maioria dos trabalhos se concentra em resultados obtidos por meio de simulações que, apesar de importantes, não representam os referidos processos em toda a sua complexidade. A grande variação da resposta destes processos, em conseqüência da característica transiente e da nãolinearidade, torna o projeto de controle clássico dispendioso e dependente de metodologia de "tentativa-e-erro".

A recuperação da enzima bromelina é um destes processos biotecnológicos, tradicionalmente operado de forma manual. A bromelina possui alto valor comercial e crescente demanda em aplicações farmacêuticas e industriais (Kelly, 1996; Freiman e Sabaa Srur, 1999).

O controle de temperatura durante todo o processo de recuperação da bromelina, por técnica de precipitação com solvente a frio, é um fator de extrema importância, pois a temperatura interfere diretamente na atividade final da enzima precipitada (Lucarini et alli, 2005). Com a utilização de controladores procura-se evitar a desnaturação da enzima, e conseqüentemente melhorar a qualidade do produto obtido.
Neste contexto, o presente trabalho descreve a aplicação prática de um sistema de automação Fieldbus ao estudo experimental de desenvolvimento e implementação de controladores convencional e Fuzzy a uma planta-piloto de precipitação de bromelina proveniente do extrato aquoso de resíduos de processamento de abacaxi. Para avaliação de desempenho dos controladores digitais foram utilizados os seguintes parâmetros: ITAE (integral of Time multiplied by Absolute of the Error), tempo de estabilização, saturação do elemento final de controle, atividade enzimática do produto e o consumo de energia elétrica do sistema de resfriamento.

\section{A PLANTA PILOTO DE PRECIPITAÇÃO}

\subsection{Descrição da Planta}

O sistema desenvolvido para os testes experimentais dos controladores Fuzzy e PID convencional foi montado no Laboratório de Controle e Automação de Processos, Faculdade de Engenharia Química, UNICAMP. A instrumentação deste sistema é representada pelo fluxograma contido na Figura 1.

Na figura 2 é apresentada a planta-piloto para operação em batelada alimentada, representda pelo fluxogragrama contido na figura 1, a qual é constituída basicamente pelos seguintes elementos: um tanque principal de aço inox com capacidade de aproximadamente $1000 \mathrm{~mL}$ (1), onde ocorre a precipitação da enzima; um tanque de armazenamento do agente de

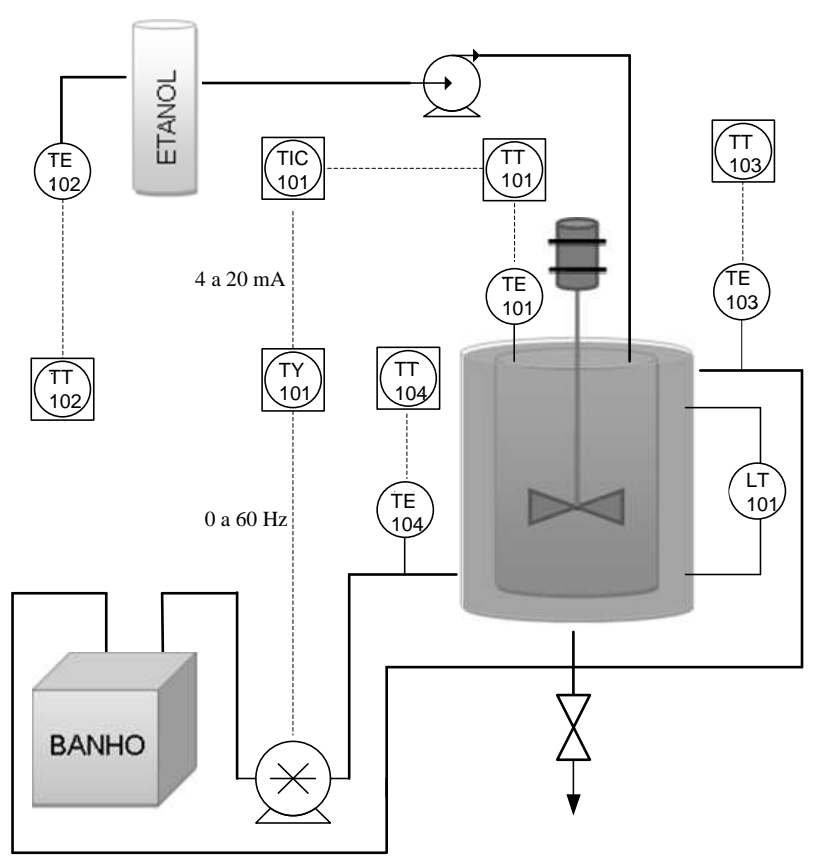

Figura 1: Fluxograma de instrumentação do sistema de precipitação. 

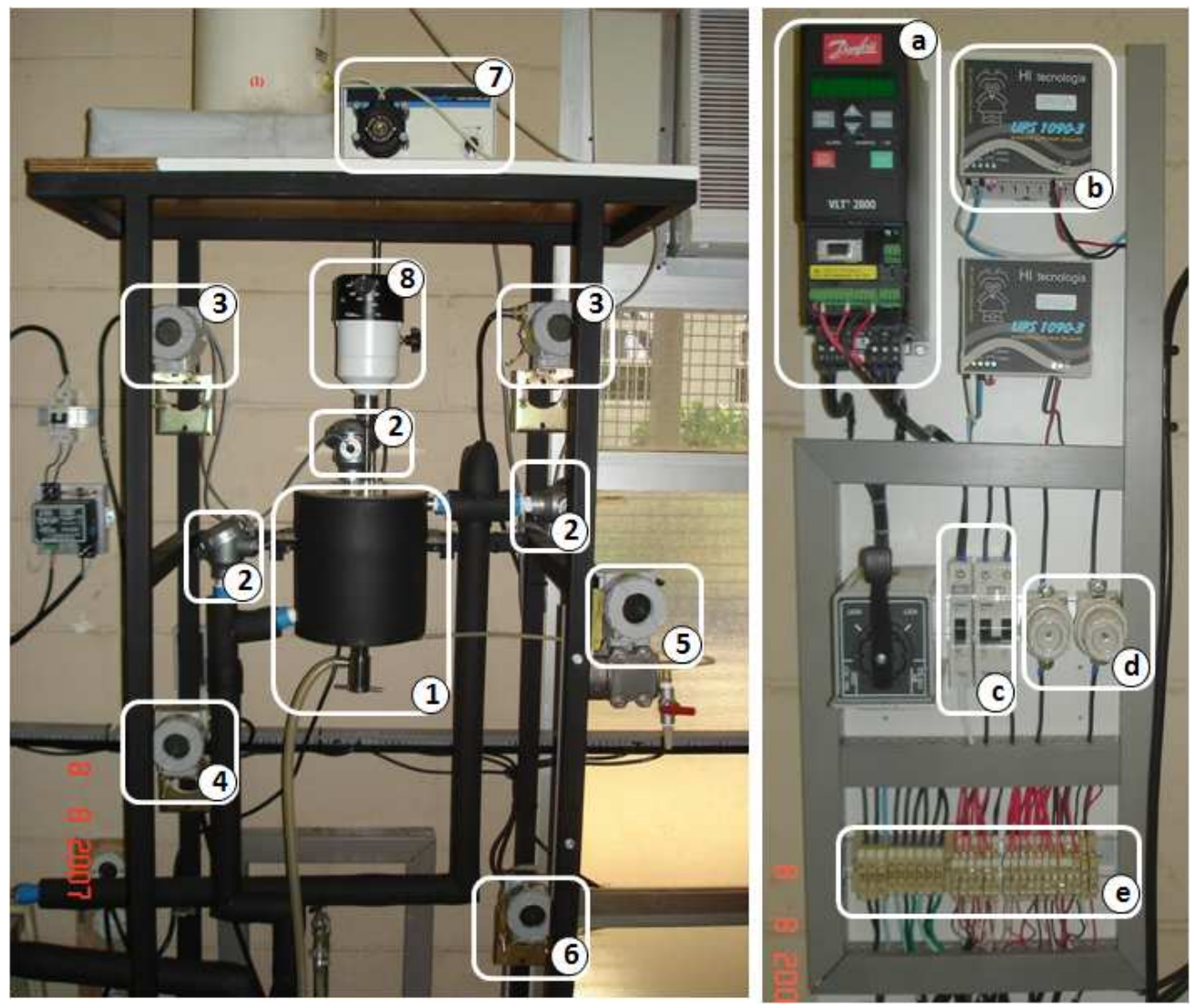

Figura 2: Sistema de precipitação da bromelina do Laboratório de Controle e Automação de Processos, Faculdade de Engenharia Química, UNICAMP.

precipitação (álcool etílico, 99,5\%); uma bomba de deslocamento positivo do fabricante RZR modelo 500, $1 \mathrm{HP} 220 \mathrm{~V}$, com vazão máxima de aproximadamente $1000 \mathrm{~L} / \mathrm{h}$, a fim de possibilitar a circulação do fluido refrigerante (propilenoglicol) pela camisa de resfriamento, apta a funcionar nas condições de alta viscosidade do fluido às baixas temperaturas; quatro termoresistências do tipo Pt 100 (2): TE101, TE102, TE103 e TE104, que possibilitam o monitoramento das temperaturas do tanque de precipitação, de entrada do álcool, da saída e da entrada da camisa de refrigeração, respectivamente; dois transmissores de temperatura fieldbus do tipo TT302 da Smar (3) com sinal de saída digital, ligados diretamente às termoresistências Pt100 para monitoramento e controle das condições operacionais do sistema; um conversor Fieldbus-corrente (FI302, Smar), para variação da rotação da bomba de fluido de resfriamento (4); um transmissor de nível (LT 101), que consiste num transmissor de pressão diferencial (LD 302) da Smar (5), que possibilitou o monitoramento do nível do tanque de precipitação da bromelina; um conversor corrente-Fieldbus (IF302, Smar), para receber o sinal do sensor de nível do tanque e a potencia consumida pela bomba do fluido de resfriamento (6); uma bomba peris- táltica da marca Masterflex Pump Controller da Cole Parmer Instrument Co. (7), para alimentação contínua do álcool etílico no tanque de precipitação; um agitador mecânico (125 rpm) para permitir homogeneização da reação (8). Tubulações de cobre soldável de $1 / 2$ in foram utilizadas, sendo revestidas com tubos de borracha elastomérica (Armaflex) a fim de evitar a perda de calor para o meio ambiente. Um painel de comandos elétrico foi montado para facilitar as ligações entre os instrumentos contidos na planta piloto. Neste painel podem ser observados: conversor de freqüência Danfoss VLT 2800 (a), que age como elemento final de controle, alterando a velocidade de funcionamento da bomba de propilenoglicol (variável manipulada); fontes (b) para alimentação elétrica dos sensores e equipamentos; dispositivos de seguranças: disjuntores (c) e fuzíveis (d); e conectores (e) para facilitar as manutenções correticas das ligações elétricas.

\subsection{Condições Operacionais da planta}

Foram preparadas alíquotas de extrato aquoso a partir da casca e do talo do abacaxi, variedade pérola, devidamente triturados com água destilada na proporção de 1:1 e filtrados. 
Em seguida, as alíquotas de $150 \mathrm{~mL}$ foram armazenadas em congelador a aproximadamente $-18{ }^{\circ} \mathrm{C}$ até serem usadas nos experimentos (Cesar et alli, 1999).

Alimentava-se o tanque de precipitação com $150 \mathrm{~mL}$ da alíquota de extrato aquoso de abacaxi e, continuamente, o etanol a temperatura ambiente, até que um volume máximo de $750 \mathrm{~mL}$ fosse obtido. Este volume corresponde à proporção de 1:4 (extrato/álcool) no tanque, visto que esta é a proporção mais adequada para promover melhor eficiência na precipitação da bromelina, segundo César et alli, 1999.

Por meio de um planejamento experimental (Leite et alli, 2008), foram determinadas as seguintes condições de operação: vazão fixa do agente de precipitação da enzima (etanol) de $0,6 \mathrm{~mL} / \mathrm{s}$; rotação inicial da bomba do fluido refrigerante propilenoglicol de $40 \%$ (374 L/h); e temperatura de entrada do propilenoglicol de $0^{\circ} \mathrm{C}$. Durante cada batelada foi mantida uma agitação constante de $150 \mathrm{rpm}$ e o tempo de contato entre o agente de precipitação e as proteínas presentes na amostra foi de 17 minutos.

\subsection{Caracterização da enzima}

Uma vez que o controle da temperatura exerce influência significativa na qualidade final da enzima recuperada no processo de precipitação da bromelina, a determinação da atividade enzimática foi utilizada como um importante índice de desempenho para a comparação da eficiência entre os controladores aplicados neste trabalho. Esta análise baseia-se no método colorimétrico de biureto. Em meio alcalino, o cobre presente no reativo de biureto reage com proteínas formando um complexo peptídico que absorve radiação UV em $540 \mathrm{~nm}$, possibilitando a análise da amostra através de espectrofotometria. De acordo com este método, uma unidade de atividade enzimática corresponde à quantidade de enzima capaz de variar em uma unidade a leitura de absorbância a $540 \mathrm{~nm}$, durante $10 \mathrm{~min}$ a $37{ }^{\circ} \mathrm{C}$. A atividade enzimática específica, $A E(U / g)$, consiste na relação entre o número de unidades de atividade enzimática $(\mathrm{U} / \mathrm{mL})$ e a quantidade de proteínas $(\mathrm{g} / \mathrm{mL})$ existentes na amostra.

\section{SISTEMA DE AUTOMAÇÃO}

A supervisão e o controle do processo foram realizados através da utilização de dispositivos e controladores que integram o sistema de automação Fieldbus (Figura 3) do Laboratório de Controle e Automação da FEQ/UNICAMP.

A rede fieldbus utilizada é composta pelos seguintes dispositivos do fabricante SMAR Equipamentos Industriais Ltda.:

- Interface de campo distribuída (DFI 302): gerenciador de comunicação, ou seja, o componente de hardware que con-

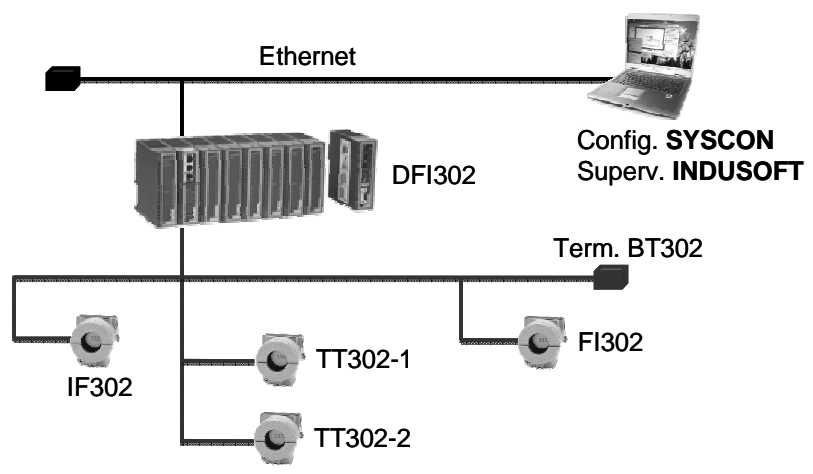

Figura 3: Rede fieldbus para o processo de precipitação de bromelina.

trola as ações relacionadas ao sistemaFieldbus, executando a maioria das funções exigidas pelo sistema de controle. O DFI conecta a rede de dispositivos de campo a uma rede Ethernet utilizando para isso um switch. Possui endereço fixo de IP, o que permite ser acessado pelo sistema supervisório através da tecnologia OPC (OLE for process control);

-Conversor corrente - fieldbus (IF 302): permite a interligação de instrumentos com saída analógica de 4 a $20 \mathrm{~mA}$ à rede Foundation Fieldbus. Possui três canais de entrada independentes. No primeiro canal foi conectado o LT301, usado para medição do nível do tanque e, no segundo canal, a saída do inversor de frequiência para acompanhamento da potência consumida pela bomba de propilenoglicol;

-Conversor Fieldbus - corrente (FI 302): converte sinais digitais Fieldbus para sinais analógicos de 4-20 mA. Neste dispositivo foi conectado o inversor de frequiência (elemento final de controle), que recebia as informações provenientes do controlador PID ou Fuzzy para alteração da vazão de resfriamento;

- Transmissores inteligentes de temperatura (TT302): estes dispositivos possuem dois canais que transformam sinais analógicos em padrão de protocoloFieldbus. No primeiro transmissor de temperatura estão ocupando os dois canais, as termoresistências: TE101 (temperatura do tanque de precipitação) e TE102 (temperatura do álcool). No segundo transmissor de temperatura ocupam os respectivos canais os sensores de temperatura: TE103 (temperatura de saída da camisa de resfriamento) e TE104 (temperatura de entrada na camisa).

A configuração dos dispositivos da rede Fieldbus e a implementação das malhas de controle convencionais foram realizadas através do software SYSCON 7.0, sendo possível realizar alterações, manutenções e operações on-line. Os principais blocos funcionais padrões (Resource, Transducer, Display, Analog Input, Analog Output, Signal Characterizer, 
PID) do software SYSCON foram devidamente configurados de maneira a se conseguir a funcionalidade desejada de cada dispositivo. Para o gerenciamento e aquisição de dados do sistema foi utilizado o software supervisório INDUSOFT Web Studio 6.1.

\section{PROJETO DOS CONTROLADORES}

\subsection{Controle Convencional PID}

Para a implementação deste controlador, utilizou-se o bloco funcional PID do software SYSCON, no qual a saída do controlador, $c(t)$, é dada pela Equação 1 .

$$
c(t)=K_{c} \varepsilon(t)+\frac{K_{c}}{\tau_{i}} \int \varepsilon(t) d t+K_{c} \tau_{d} \frac{d \varepsilon}{d t}+c_{s}
$$

onde o erro é dado por $\varepsilon(t)=Y(t)-Y_{S P}(t)$, sendo $Y(t)$ o valor medido da variável controlada e $Y_{S P}$ o seu set-point, no instante de tempot. O parâmetros ajustáveis são: o ganho estático do controlador, $K c$; a constante de tempo integral, $\tau_{i}$; e a constante de tempo derivativo, $\tau_{d}$. A constante $c_{s}$ corresponde ao valor da variável manipulada quando o erro é nulo (rotação da bomba $=40 \%$ ).

Visando a obtenção da curva de reação do processo, uma condição de estado pseudo-estacionário foi simulada experimentalmente através da adição do extrato aquoso de abacaxi com vazões iguais de entrada e saída no tanque de precipitação, de forma a manter o volume constante. A adição do etanol se iniciava quando o sistema encontrava-se a uma temperatura de $5^{\circ} \mathrm{C}$ e, neste mesmo instante, era realizada uma perturbação na bomba de propilenoglicol de $30 \%$ de sua capacidade máxima, a partir da condição inicial de $40 \%$. Neste processo de sintonia, o extrato de abacaxi utilizado foi diluído com etanol (1:1) para manter as mesmas condições da etapa inicial da precipitação em batelada. Os dados foram monitorados até que a temperatura de reação alcançasse um novo estado estacionário, permitindo estimar os valores dos parâmetros do processo: $K_{p}$, ganho estático; $\tau_{p}$, constante de tempo; e $\mathrm{t}_{d}$, tempo morto. De posse dos valores destes parâmetros, o método de Ziegler-Nichols foi aplicado para a sintonia do controlador convencional, resultando em estimativas iniciais dos parâmetros do controlador: $K_{c}, \tau_{i}$ e $\tau_{d}$. Entretanto, mesmo seguindo esta metodologia clássica, os parâmetros estimados não se mostraram adequados e, portanto, seguiuse uma exaustiva sequência de experimentos para adequação destes parâmetros por tentativa-e-erro, tendo-se por base os seguintes índices de desempenho: ITAE, tempo de estabilização e saturação do elemento final de controle.

\subsection{Controle Fuzzy}

A idéia básica do controle Fuzzy é modelar as ações a partir de conhecimento especialista, diferindo dos métodos convencionais de controle de processos químicos que normalmente empregam a modelagem matemática fenomenológica baseada em balanços de massa, energia e momento, além das leis dos fenômenos de transporte, cinética e termodinâmica.

Uma vez que o desenvolvimento do controlador Fuzzy necessita do conhecimento especialista para observação do comportamento do sistema, seis ensaios experimentais foram realizados utilizando diferentes volumes fixos (300, 450 e 600 $\mathrm{mL}$ ) no tanque de precipitação, os quais correspondem a proporção de 1:1 a 1:3 extrato de abacaxi/etanol. Da mesma forma que na sintonia do controlador PID, foram simuladas condições de estado pseudo-estacionário, sendo que, para cada uma das três condições de volume, o sistema foi submetido a perturbações de $\pm 30 \%$ na rotação da bomba de propilenoglicol. Através das curvas de reação geradas foi possível avaliar a influência da alteração do volume no processo de precipitação. As observações obtidas a partir desta etapa contribuíram para o desenvolvimento do controlador Fuzzy.

No presente trabalho foi utilizado o controlador Fuzzy do tipo Mamdani, como proposto por Li e Gatland (1996). As 49 regras originais propostas estão apresentadas na Tabela 1. Os termos lingüísticos utilizados nas funções de pertinência, das variáveis de entrada e saída, foram: GP (grande positivo), MP (médio positivo), PP (pequeno positivo), ZR (zero), GN (grande negativo), MN (médio negativo), e PN (pequeno negativo).

O erro $(\varepsilon)$ e a variação do erro $(\Delta \varepsilon)$ foram utilizados como variáveis de entrada deste controlador incremental. A variação da rotação da bomba de propilenoglicol foi utilizada como variável de saída do controlador, definida como $\Delta \mathrm{U}(\mathrm{t})$. A estrutura do controlador Fuzzy-PI utilizado está representada na Figura 4.

Tabela 1: Regras de Mamdani (Li e Gatland, 1996).

\begin{tabular}{|c|c|c|c|c|c|c|c|}
\hline \multirow{2}{*}{ ERRO $(\varepsilon)$} & \multicolumn{7}{|c|}{$\Delta$ ERRO $(\Delta \varepsilon)$} \\
\cline { 2 - 7 } & GN & MN & PN & ZR & PP & MP & GP \\
\hline GP & ZR & PP & MP & GP & GP & GP & GP \\
\hline MP & PN & ZR & PP & MP & GP & GP & GP \\
\hline PP & MN & PN & ZR & PP & MP & GP & GP \\
\hline ZR & GN & MN & PN & ZR & PP & MP & GP \\
\hline PN & GN & GN & MN & PN & ZR & PP & MP \\
\hline MN & GN & GN & GN & MN & PN & ZR & PP \\
\hline GN & GN & GN & GN & GN & MN & PN & ZR \\
\hline
\end{tabular}




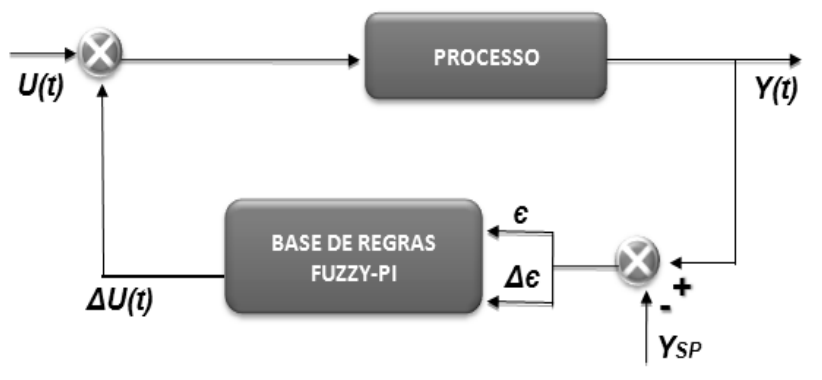

Figura 4: Estrutura do Controlador Fuzzy-PI.

O controlador Fuzzy-PI foi desenvolvido utilizando-se o Toolbox Fuzzy do MATLAB 7.0.1. Foi adotada a estratégia MISO, operador de agregação min (Equação 2) e centro-degravidade como critério de defuzzificação (Equação 3).

$$
\begin{gathered}
(\forall x \in X) \Rightarrow \mu_{A \cap B}(x)=\min \\
\Delta U=\frac{\sum_{i=1}^{N} u_{i} \mu_{O U T}\left(u_{i}\right)}{\sum_{i=1}^{N} \mu_{O U T}\left(u_{i}\right)}
\end{gathered}
$$

onde $\mu_{O U T}\left(u_{i}\right)$ é a área de uma função de pertinência modificada pelo resultado da inferência fuzzy e $u_{i}$ é a posição do centróide da função de pertinência individual. Tal equação calcula o centróide composto, para o qual contribuem todas as funções de pertinência indicadas.

\section{RESULTADOS}

\subsection{Determinação do Set-point}

A fim de evitar a desnaturação da proteína, a temperatura de set-point foi determinada a partir de ensaios experimentais de atividade enzimática para analisar a qualidade da enzima. Os resultados mostraram uma atividade de $0,8739 \mathrm{U} / \mathrm{mL}$ a $5^{\circ} \mathrm{C}, 0,7478 \mathrm{U} / \mathrm{mL}$ a $10^{\circ} \mathrm{C}$ e 0,7204 a $20^{\circ} \mathrm{C}$ (Figura 5), o que comprova que em temperaturas menores o efeito da desnaturação é atenuado. Sendo assim, o set-point selecionado para a temperatura foi de $5^{\circ} \mathrm{C}$, conforme temperatura utilizada no trabalho de Cesar et alli,1999.

\subsection{Projeto dos controladores}

\section{PID}

Conforme procedimento descrito no Item 4.1, obtiveram-se da curva "1:1 perturbação negativa" da Figura 6 os parâme-

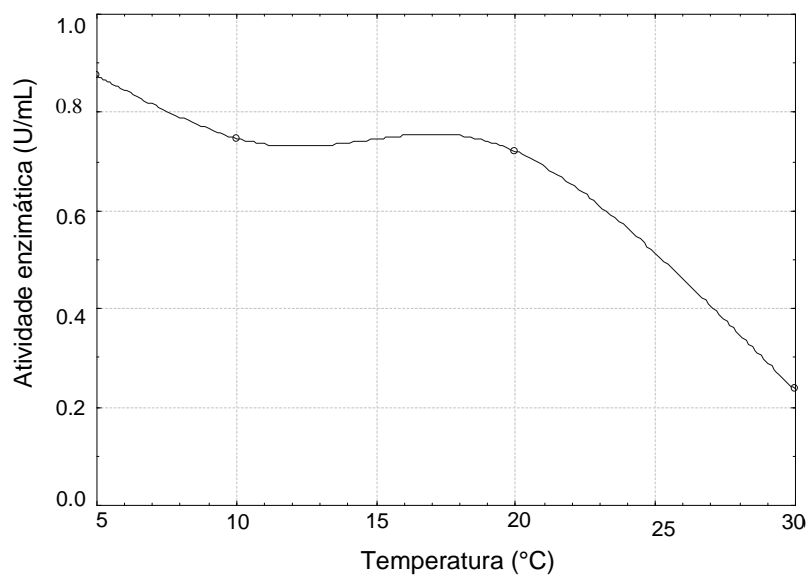

Figura 5: Influência da temperatura na recuperação da atividade enzimática.

tros da curva de reação do processo e calcularam-se os parâmetros do controlador pelas equações de sintonia de ZieglerNichols: $\mathrm{Kc}=280 \% /{ }^{\circ} \mathrm{C}$, $\tau_{i}=40 \mathrm{~s} \mathrm{eg} \tau_{d}=10 \mathrm{~s}$. Esta sintonia resultou em valor de ganho muito grande e, consequentemente, houve saturação prolongada nos limites inferior e superior da bomba de resfriamento. Portanto, ensaios em malha fechada foram realizados reduzindo-se principalmente este parâmetro. Seguiramse inúmeros ensaios para a determinação da combinação de parâmetros $\left(K_{c}, \mathrm{~g} \tau_{i} \operatorname{eg} \tau_{d \mathrm{~g}}\right)$ mais adequada para a atuação do controlador PID. Os melhores parâmetros encontrados, após a sintonia fina deste controlador por tentativa-e-erro, foram: $\mathrm{K}_{c}=35 \% /{ }^{\circ} \mathrm{C}, \tau_{i}=28 \mathrm{~s} \mathrm{eg} \tau_{d}=7 \mathrm{~s}$. Os resultados em malha fechada são encontrados no item 5.3.

\section{Fuzzy}

A sintonia do controlador Fuzzy baseou-se na observação das curvas de reação do tanque de precipitação frente a perturbações positivas e negativas de intensidade $30 \%$ na rotação da bomba de fluido de resfriamento (Figura 6), conforme procedimento descrito no item 4.2. As condições iniciais dos ensaios eram mantidas em $40 \%$ de rotação da bomba e $5^{\circ} \mathrm{C}$ para a temperatura interna do tanque.

Num sistema linear, as respostas apresentadas para perturbações opostas de igual amplitude são simétricas.

Analisando-se as curvas de reação a diferentes volumes (Figura 6), constata-se que o sistema em estudo apresenta nãolinearidades, pois os ganhos estáticos do processo, em módulo, aumentam à medida que aumenta o volume para condição de elevação de rotação da bomba (perturbações positivas) e os mesmos ganhos estáticos diminuem à medida que aumenta o volume para diminuição de rotação da bomba (perturbações negativas). 


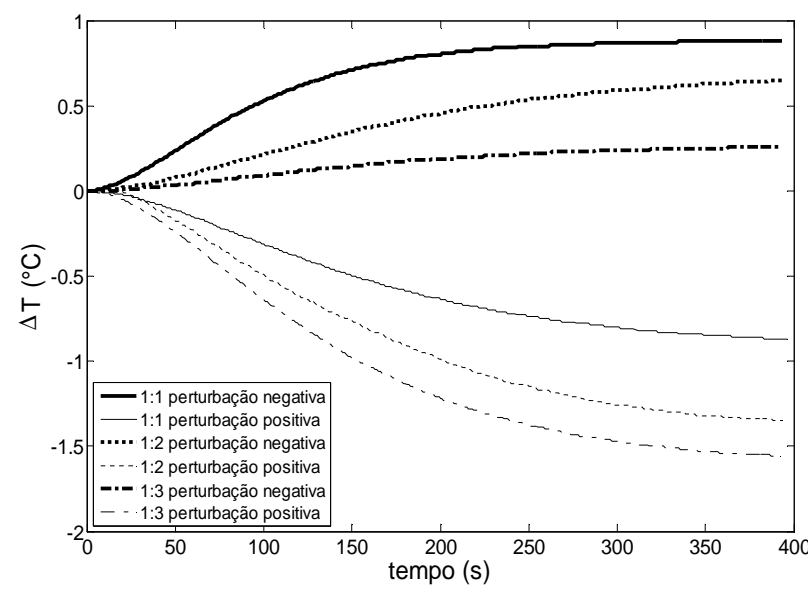

Figura 6: Curvas de reação obtidas a partir de perturbações aplicadas na variável manipulada.

A Tabela 2 resume os valores de ganho estático extraídos da Figura 6.

Tabela 2: Ganhos estáticos do processo, $\mathrm{K}_{p}$, obtidos das curvas de reação.

\begin{tabular}{|c|c|c|c|}
\hline \multicolumn{2}{|c|}{ Perturbação: } & Positiva & Negativa \\
\hline $\begin{array}{c}\text { Volume } \\
(\mathrm{mL})\end{array}$ & Proporção & $\mathrm{K}_{p}\left({ }^{\circ} \mathrm{C} / \%\right)$ & $\mathrm{K}_{p}\left({ }^{\circ} \mathrm{C} / \%\right)$ \\
\hline 300 & $1: 1$ & $-0,030$ & 0,030 \\
\hline 450 & $1: 2$ & $-0,047$ & 0,020 \\
\hline 600 & $1: 3$ & $-0,053$ & 0,010 \\
\hline
\end{tabular}

Dois efeitos principais explicam o formato das curvas de reação obtidas: a variação da área de troca térmica no tanque durante a batelada e o calor de dissolução do etanol em uma solução aquosa contendo diferentes proporções deste álcool.

Quando aplicada uma perturbação positiva na variável manipulada (rotação aumentou), na condição de volume de 600 mL (curva 1:3), a temperatura interna do tanque sofreu maior diminuição quando comparada com as condições de volume de $450 \mathrm{~mL}$ e $300 \mathrm{~mL}$. Este comportamento pode ser facilmente explicado, pois as ações de controle serão mais efetivas quanto maior o volume interno do tanque, devido a um aumento da área de troca térmica do fluido processado (extrato de abacaxi + álcool) com o fluido refrigerante. Adicionalmente, nesta condição de volume de $600 \mathrm{~mL}$, a proporção entre extrato e álcool já seria de 1:3 em volume e, portanto, menor é o distúrbio na temperatura provocado pela dissolução do etanol na solução contida no tanque. Sendo assim, nota-se que a resposta à perturbação negativa de mesma intensidade é totalmente assimétrica, pois nesta condição pra- ticamente não há fonte de energia para aumentar a temperatura.

Na condição de volume $300 \mathrm{~mL}$ e proporção 1:1, se observa um menor ganho do processo frente ao aumento de rotação da bomba, decorrente de uma deficiente troca térmica (menor área de troca). Nestas condições que simulam o início da batelada, o calor de dissolução desprendido é maior, e o ganho da perturbação negativa (rotação pequena) é o maior obtido dentre as diferentes condições de volume. Com o decorrer da precipitação esse efeito vai se tornando cada vez menor.

Com estas atribuições fortemente transientes e não-lineares, o sistema se caracteriza por possuir diferentes sensibilidades às ações de controle, o que reforça a limitação do uso dos controladores convencionais neste sistema.

Visando atenuar a conseqüência das não-linearidades do sistema, a sintonia do controlador Fuzzy consistiu na mudança nas disposições das funções de pertinência para entradas e saídas dos controladores, modificação na base de regras e ainda no universo de discurso. A determinação dos universos de discurso e disposição das funções de pertinência foram adotadas principalmente a partir das contribuições obtidas através da análise do comportamento dinâmico do processo. No entanto, a adaptação destes parâmetros foi fortemente influenciada por conhecimentos subjetivos baseados em observações práticas do comportamento do controlador diante de modificações nos mesmos.

As regras de Mamdani , apresentadas na Tabela 1, foram modificadas em função de testes realizados experimentalmente, para propiciar melhor sintonia do controlador Fuzzy e encontram-se destacadas na Tabela 3.

Para os casos em que o erro é grande positivo (GP) - temperatura está acima do set-point - e a variação do erro indica que a temperatura está diminuindo ( $\mathrm{GN}, \mathrm{MN}, \mathrm{PN})$, não foi constatada a necessidade de atuação positiva da bomba de fluido de resfriamento, como sugerida pela tabela original de regras (Tabela 1). Neste momento da batelada, a capacidade de troca térmica já é mais eficiente, devido ao aumento do volume no tanque, e também é menor o impacto de dissolução do etanol no meio de precipitação. Desta forma, as ações foram modificadas para PN, visando não saturar a ação de controle no limite superior. Além disto, a observação prática do comportamento do processo fez perceber que mesmo uma redução sutil na vazão de resfriamento seria suficiente para diminuir a temperatura e ainda evitar que a mesma não se desviasse significativamente do set-point.

Quando a temperatura está um pouco acima da desejada (erro $\mathrm{PP})$, e está aumentando com o tempo ( $\triangle \mathrm{ERRO}$ GP), há a necessidade de atuação positiva da bomba de refrigeração. No entanto, como o erro ainda era pequeno, observou-se que não 
havia necessidade de uma atuação grande (GP), pois a atuação média positiva (MP) cumpriria o mesmo objetivo e ainda evitaria oscilações da temperatura ao redor do set-point.

Tabela 3: Matriz de base de regras modificadas para controlador Fuzzy-PI.

\begin{tabular}{|c|c|c|c|c|c|c|c|}
\hline \multirow{2}{*}{ ERRO } & \multicolumn{7}{|c|}{$\Delta$ ERRO } \\
\cline { 2 - 8 } & GN & MN & PN & ZR & PP & MP & GP \\
\hline GP & $\underline{\text { PN }}$ & $\underline{\text { PN }}$ & $\underline{\text { PN }}$ & GP & GP & GP & GP \\
\hline MP & PN & ZR & PP & MP & GP & GP & GP \\
\hline PP & MN & PN & ZR & PP & MP & GP & $\underline{\text { MP }}$ \\
\hline ZR & GN & MN & PN & ZR & PP & MP & GP \\
\hline PN & GN & GN & MN & PN & ZR & PP & $\underline{\text { PP }}$ \\
\hline MN & GN & GN & GN & MN & PN & ZR & $\underline{\mathbf{Z R}}$ \\
\hline GN & GN & GN & GN & GN & MN & PN & ZR \\
\hline
\end{tabular}

No caso em que a temperatura está um pouco abaixo da desejada (erro PN), no entanto, se eleva rapidamente ( $\triangle \mathrm{ERRO}$ GP), para conter esta elevação observou-se que uma atuação pequeno positivo (PP) da bomba seria suficiente, uma vez que o ganho do processo é maior nestas condições finais da batelada para perturbações positivas.

A atuação originalmente sugerida de médio positivo (MP) na bomba poderia propiciar uma redução significativa da temperatura e provocar oscilações. Pelas mesmas razões, optou-se em não atuar na bomba ( $\Delta U \mathrm{ZR})$, na situação de erro médio negativo (MN) e variação grande positiva (GP), uma vez que a manutenção da vazão daquele instante seria suficiente para evitar qualquer elevação de temperatura. As funções de pertinência triangulares, determinadas após a sintonia deste controlador, estão representadas na Figura 7.

Pode-se observar a definição de uma estreita faixa (-1 a 1) para o universo de discurso do erro $(\varepsilon)$, devido ao fato de o início do processo ser a etapa mais crítica, onde é exigida uma rápida atuação da variável manipulada, mesmo para pequenos desvios da variável controlada. Isto também explica o uso da ampla faixa $\left(-20\right.$ a $\left.13^{\circ} \mathrm{C}\right)$ adotada para o universo de discurso da variável de saída $\Delta \mathbf{U}$. A escala negativa (-20), em termos de valores absolutos, foi definida maior que a positiva (13) devido à observação da necessidade de frear mais rapidamente o resfriamento após a etapa inicial, onde menores vazões de propilenoglicol são capazes de conter a elevação da temperatura do tanque provocada pela menor liberação de calor de dissolução do álcool deste estágio, conforme conhecimento obtido na etapa de verificação das curvas de reação do processo.

A superfície de controle gerada pelo controlador Fuzzy-PI desenvolvido é apresentada na Figura 8, onde podem ser observadas as não linearidades embutidas neste tipo de controlador.

\subsection{Ensaios em malha fechada}

As Figuras 9 e 10 apresentam, respectivamente, o comportamento do sistema sob ação dos controladores PID convencional e Fuzzy-PI, sintonizados conforme configurações apresentadas no Item 5.2. Estes ensaios de precipitação em malha fechada foram realizados utilizando as mesmas condições experimentais de processo. Na Tabela 4 estão resumidos os índices de desempenho calculados a partir dos comportamentos obtidos nas Figuras 9 e 10.

De acordo com a Tabela 4, observa-se que a atividade enzimática específica, forte indicador da qualidade do produto, é maior quando o controlador Fuzzy foi utilizado. Este fato deve-se a menor flutuação da temperatura interna do tanque, com menor tempo de estabilização, quando se utilizou o controlador Fuzzy. Desta forma o efeito de desnaturação da bromelina é reduzido, proporcionando melhor qualidade da enzima, uma vez que esta apresenta sensibilidade à variação de temperatura. A concentração média de proteínas totais na amostra final após o processo de precipitação foi de 0,8358 $\pm 0,0194 \mathrm{mg} / \mathrm{mL}$ em $\mathrm{pH} 7,0$.

Nota-se também que o controlador Fuzzy proporcionou desempenho mais adequado da variável manipulada, quando comparado ao controlador convencional PID: o tempo de saturação da bomba de propilenoglicol diminuiu de 130 para 35 $\mathrm{s}$, reduzindo o consumo de energia de 9,11 para $3,65 \mathrm{kWh}, \mathrm{o}$ que representa uma economia de energia elétrica de 59,93\%. Esta redução significativa no consumo de energia elétrica para o controlador Fuzzy é consequência de um ajuste mais adequado das funções de pertinência na região em que o erro é "grande positivo" e variação do erro é "grande negativo".

Menor valor do parâmetro de desempenho ITAE foi apresentado pelo uso do controlador Fuzzy. Esse comportamento pode ser explicado pela reduzida amplitude de oscilação da variável controlada em torno do valor de referência, durante a maior parte do ensaio, a qual determinou desempenho superior deste controlador.

\section{CONCLUSÕES}

Este trabalho apresentou o desenvolvimento e a aplicação experimental de controladores Fuzzy e PID convencional em um processo de precipitação da bromelina, com o objetivo de realizar controle automático de temperatura a fim se evitar o efeito de desnaturação da enzima. A operação em malha aberta, com valor pré-fixado da rotação da bomba de fluido refrigerante, mostrou-se ineficiente, com elevado overshoot, 


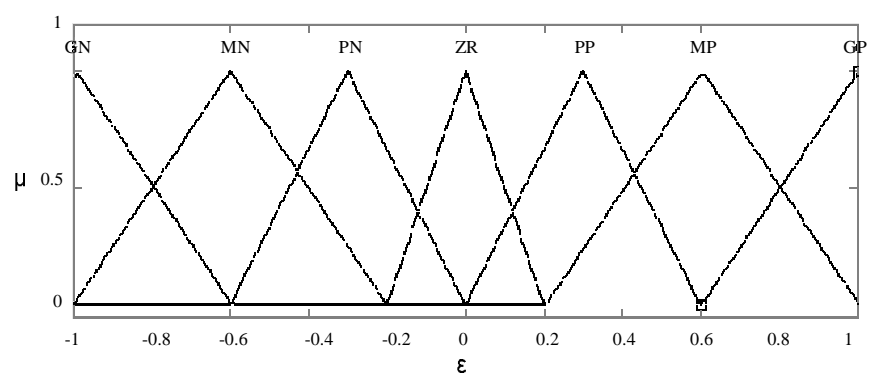

(a)

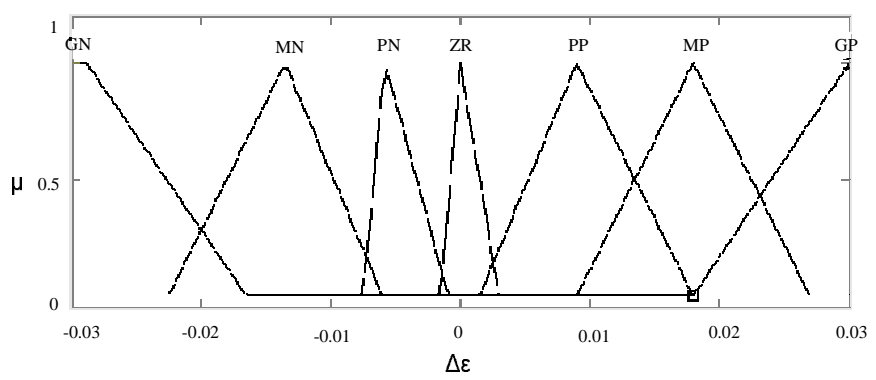

(b)

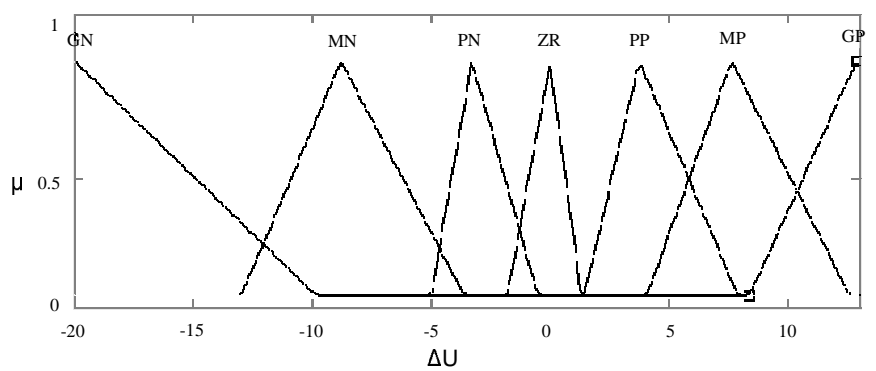

(c)

Figura 7: Estruturas das funções de pertinência para as variáveis: (a) Erro $(\varepsilon)$; (b) Variação do erro $(\Delta \varepsilon)$; (c) variação de saída $(\Delta U)$ empregadas no desenvolvimento do controlador fuzzy-PI.

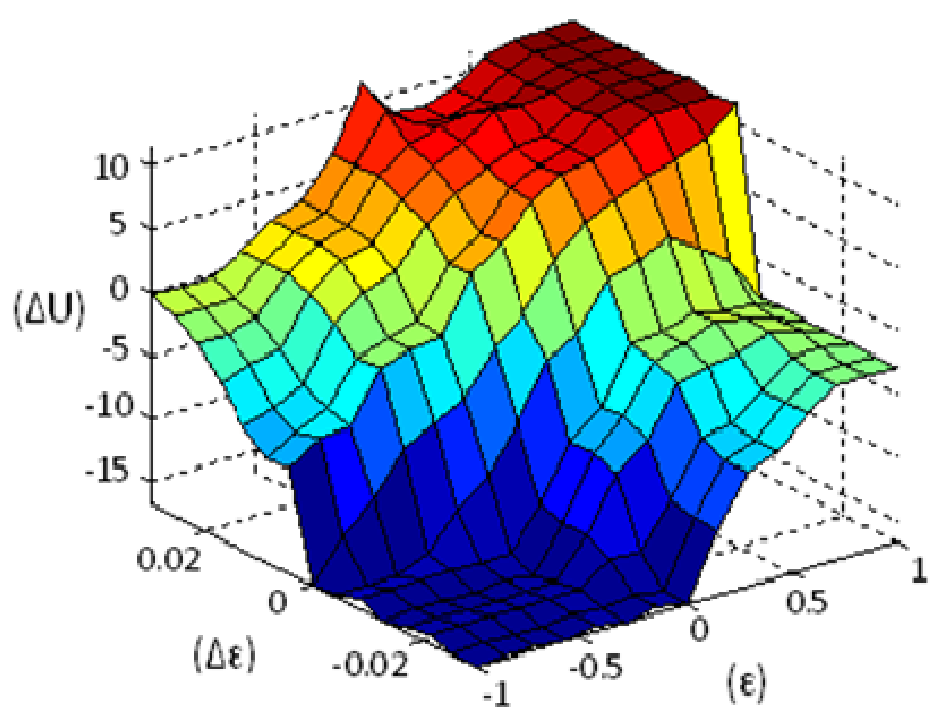

Figura 8: Superfície de controle do controlador Fuzzy-PI. 
Tabela 4: Índices de desempenho comparativos entre os ensaios de controle.

\begin{tabular}{|c|c|c|c|}
\hline & $\begin{array}{c}\text { Malha } \\
\text { Aberta }\end{array}$ & PID $_{2}$ & Fuzzy \\
\hline${\text { Overshoot }\left({ }^{\circ} \mathbf{C}\right)}$ ) & 5 & 3,1 & 3,1 \\
\hline Tempo de resposta (s) & 281 & 171 & 171 \\
\hline Tempo de estabilização (s) & $*$ & 400 & 171 \\
\hline Tempo de saturação da bomba (s) & $*$ & 130 & 35 \\
\hline ITAE (x10 ${ }^{3}$ ) & 950,5 & 80,3 & 56,6 \\
\hline Atividade enzimática especifica (U/g) & 0,32 & 1,03 & 1,80 \\
\hline Energia consumida (kWh) & 42 & 9,11 & 3,65 \\
\hline
\end{tabular}
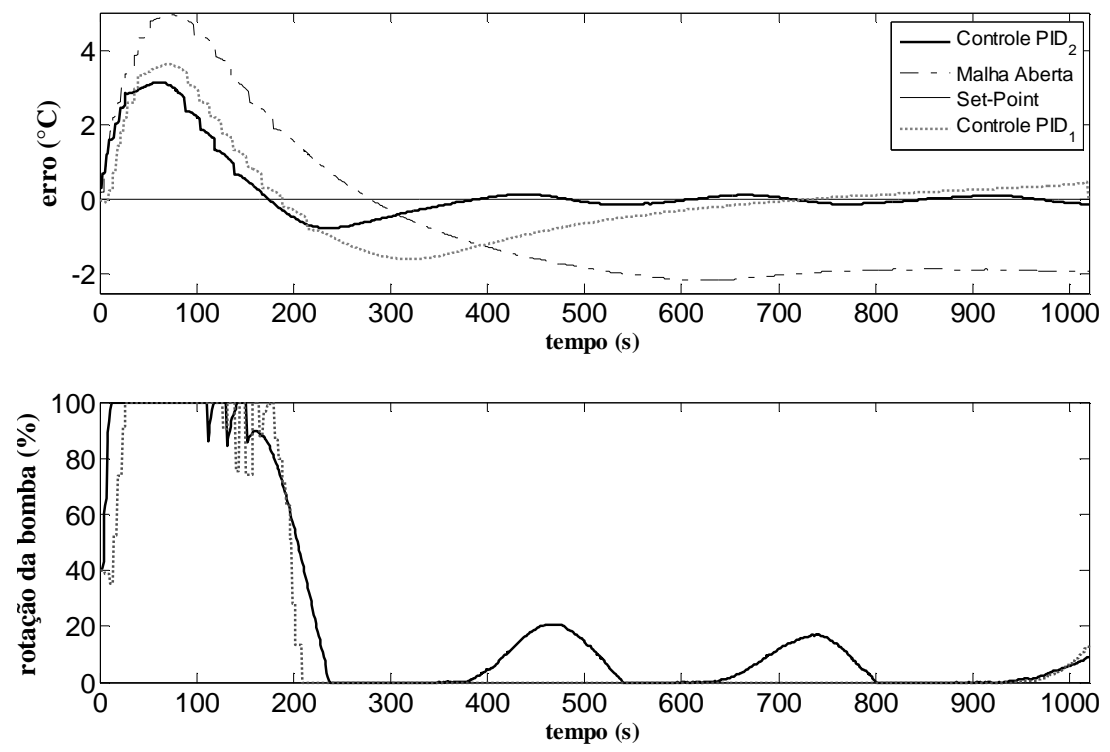

Figura 9: Comportamento da variável controlada e manipulada, sob a ação de controladores PID com as seguintes sintonias: $\mathrm{PID}_{1}\left(\mathrm{~K}_{c}=8 \% /^{\circ} \mathrm{C}, \tau_{i}=28,0\right.$ s eg $\left.\tau_{d}=1,5 \mathrm{~s}\right)$ e $\mathrm{PID}_{2}\left(\mathrm{~K}_{c}=35 \% /{ }^{\circ} \mathrm{C}, \tau_{i}=28,0 \mathrm{~s}\right.$ eg $\left.\tau_{d}=7,0 \mathrm{~s}\right)$.

distanciamento do valor de set-point e, consequentemente, baixa atividade enzimática do produto final.

Diante dos resultados apresentados, pode-se concluir que o controlador Fuzzy apresentou desempenho superior para o controle da temperatura do processo, quando comparado com o controlador convencional. Dentre as melhorias significativas, ressalta-se: menor valor de ITAE, menor tempo de estabilização, melhor atuação da variável manipulada e maior recuperação de atividade enzimática (74,75\%). Apresentou ainda menor consumo de energia elétrica $(59,93 \%)$, proporcionando assim significativa redução de custos de operação.

A maior eficiência do controlador Fuzzy pode ser atribuída a sua adaptação às não-linearidades comprovadas do sistema, apresentando comportamentos mais adequados que o controlador convencional. Seguindo a estratégia de sintonia do controlador Fuzzy-PI a partir da observação de diferentes curvas de reação do processo, este controlador se apresentou como a opção mais atrativa para o controle sistema de precipitação da bromelina.

O sistema de controle em rede Fieldbus, que alimentou a troca de informações entre os softwares INDUSOFT e MATLAB e os instrumentos de campo em tempo real, se mostrou confiável e rápido para a aplicação em questão.

\section{AGRADECIMENTOS}

\section{CAPES e CNPq}



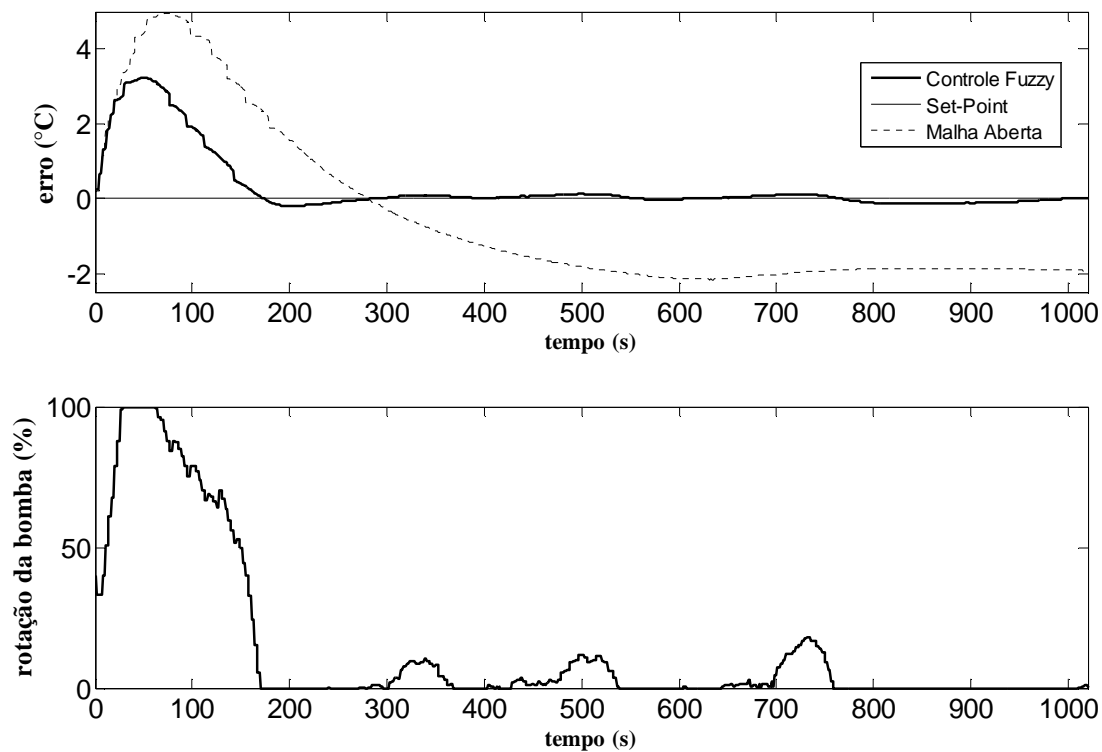

Figura 10: Comportamento da variável controlada e manipulada, sob a ação do controlador Fuzzy-PI.

\begin{tabular}{|l|l|}
\hline \multicolumn{2}{|c|}{ Nomenclatura } \\
\hline$\epsilon=$ erro; & MISO = multiple-input single-output; \\
\hline$\Delta \epsilon=$ variação do erro; & $u_{i}=$ função de pertinência (nível de atuação); \\
\hline$\Delta T=\epsilon ;$ & $i=$ índice; \\
\hline$K_{c}=$ ganho do controlador; & $U(t)=$ ação de controle \\
\hline$\tau_{d}=$ tempo derivativo; & $\Delta U(t)=$ variação da ação de controle \\
\hline$\tau_{i}=$ tempo integral; & $N=$ função de pertinência negativa; \\
\hline$c_{s}=$ valor da variável manipulada quando $\epsilon=0 ;$ & $P=$ função de pertinência positiva; \\
\hline$Y_{S P}=$ "set-point" (valor de referência); & OPC = controle de processos por "OLE" \\
\hline$Y(t)=$ saída do processo; & OLE = Object Linking and Embedding \\
\hline
\end{tabular}

\section{REFERÊNCIAS}

Antunes, A.J.B., Pereira, J.A.F.R., Fileti, A.M.F. (2005), Fuzzy control of a PMMA batch reactor: development and experimental testing. Computres and Chemical Engineering, 30, 268-276.

Babuska, R., Oosterhoff, J., Oudshoorn, A., e Bruijim, P.M. (2002). Fuzzy self-tuning PI control of $\mathrm{pH}$ in fermentation. Engineering Applications of Intelligence Artificial, 15, 3-15.

César, A.C.W., Silva, R., e Lucarini, A.C. (1999). Pineapple stem and rind proteolytic enzymes recovery (In Portuguese). Revista de Iniciação Científica - São Carlos/SP,1, 47-53.

Fileti, A.M.F., Antunes, A.J.B., Silva, F.V., e Silveira Jr, V. (2007). Experimental investigations on fuzzy logic for process control. Control Engineering Practice, 15, 1149-1160.

Freiman, L.O., e Sabaa Srur, A.U.O. (1999). Determination of total protein and aminoacid composition of bromelain extracted from pineapple plant residues (Ananás Comosus, (L.) Merril). Ciência e Tecnologia de Alimentos. 19(2). ISSN 0101-2061.

Horiuchi, J., e Kishimoto, M. (2002). Application of Fuzzy Control to Industrial Bioprocess in Japan. Fuzzy sets and Systems, 128, 117-124.

Kelly, G. S. (1996). Bromelain: A literature review and discussion of its therapeutic applications. Alternative Medicine Review, 1(4): 243-257.

Lee, C.C. (1990). Fuzzy logic control systems: fuzzy logic controllers_Part I. IEEE Transactions on Systems, Man and Cybernetics,20(2), 404-418. 
Leite, M. S.; Fujiki, T. L.; Fileti, A. M. F.; Silva, F. V. Determinação das condições operacionais de um processo de precipitação da Bromelina via planejamento experimental. In: XVII COBEQ, 2008, Recife. Anais do XVII COBEQ. Recife, 2008. v. CD. p. 869.

Li, H. e Gatland, H.D. (1996). Conventional fuzzy control and its enhancement. IEEE Transactions on Systems, Man and Cybernetics_part B: Cybernetics, 26(5), 791797.

Lucarini, A.C., Kilikian, B.V., e Pessoa Jr. (2005). Purificação de produtos biotecnológicos. Manole: São Paulo.

Martìnez, G., López, A., Esnoz, A., Vírseda, P., e Ibarrola, J. (1999). A new fuzzy control system for white wine fermentation. Food Control, 10, 175-180.

Pagliosa, A.L. (2003). Obtenção das funções de pertinência de um sistema neurofuzzy modificado pela rede de Kohonen. Dissertação de Mestrado, Universidade do Estado de Santa Catarina, UDESC, Joinville-SC.

Sandri, S. e Correa C. (1999). Lógica Nebulosa. V Escola de Redes Neurais: Conselho Nacional de Redes Neurais, São José dos Campo S.P., pp.c-073-c090.

Shaw, I.S. e Simões, M.G. (1999). Controle e Modelagem Fuzzy. FAPESP, Edgar Bluchner, São Paulo.

Silva, F.V., Neves Filho, L.C., Silveira Jr, V. (2006). Experimental investigation of fuzzy controller for the temperature control of the secondary refrigerant in a liquid chiller. Journal of Food Engineering, 75, 349-354.

Souza Jr., e Almeida, P.I.F. Design of a fuzzy system for the control of a biochemical reactor in fed-batch culture. Process Biochemistry, 37, 461- 469.

Steyer, J.P., Estaben, M., e Polit, M. (1997). Fuzzy control of an anaerobic digestion process for the treatment of an industrial wastewater. IEEE, Fuzzy Systems, 3 (1-5), 1245-1249. 